Kopfischmerz News der DMKG

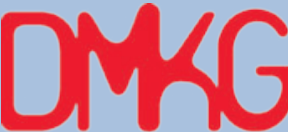

Deutsche Migräne- und Kopfschmerzgesellschaft

\title{
Achtsamkeitsbasierte Stressreduktion zur prophylaktischen Behandlung der episodischen Migräne: Eine randomisiert kontrollierte Studie
}

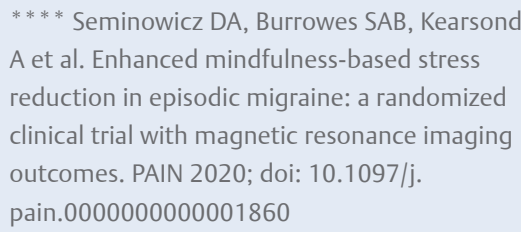

**** Seminowicz DA, Burrowes SAB, Kearsond $A$ et al. Enhanced mindfulness-based stress reduction in episodic migraine: a randomized clinical trial with magnetic resonance imaging outcomes. PAIN 2020; doi: 10.1097/j. pain. 0000000000001860

Achtsamkeitsbasierte Stressreduktion (MBSR) zur prophylaktischen Migränebehandlung bei Erwachsenen ist effektiv und teilweise wirksamer als ein herkömmliches Stressbewältigungstraining.

\section{Hintergrund}

Achtsamkeits- und akzeptanzbasierte Therapieverfahren wie z. B. die achtsamkeitsbasierte Stressreduktion („mindfulnessbased stress reduction “, MBSR) [1] oder die achtsamkeitsbasierte kognitive Therapie ( "mindfulness-based cognitive therapy“, $\mathrm{MBCT}$ ) [2] lassen sich als „Dritte Welle“ der Verhaltenstherapie verorten [3]. Diese Ansätze sind aus der klinischen Praxis und Forschung nicht mehr wegzudenken. Es liegen zahlreiche Studien zur Wirksamkeit bei den unterschiedlichsten Störungsbildern vor, das Anwendungsspektrum reicht von psychischen Erkrankungen (z. B. Angst- und affektive Störungen) bis hin zur unterstützenden Behandlung von Krebserkrankungen, Bluthochdruck oder Asthma. Die Befunde zur Evidenz sind sehr heterogen. Es finden sich teilweise leichte bis mittlere Effekte, wobei MBSR der „klassischen“ kognitiven Verhaltenstherapie in der Regel nicht überlegen ist. Zur Wirksamkeit von MBSR bei Kopfschmerz respektive bei Migräne liegen kaum Studien vor.

\section{Zusammenfassung}

In einer randomisiert-kontrollierten Studie wurde mit 98 Erwachsenen mit episodischer Migräne ein MBSR-Programm (Interventi- onsgruppe) oder ein herkömmliches Stressbewältigungstraining („stress management for headache“, SMH, aktive Kontrollgruppe) durchgeführt. Die Interventionen fanden im Gruppensetting statt und erstreckten sich jeweils über einen Zeitraum von 16 Wochen (erste 8 Wochen eine Sitzung pro Woche, zweite 8 Wochen eine Sitzung alle 2 Wochen), es wurden jeweils 12 Sitzungen zu je 2 Stunden durchgeführt. Durch die relativ lange Interventionsdauer sollte den Teilnehmern eine verbesserte Übungs- und Anwendungspraxis im Alltag ermöglich werden, woraus die Autoren das Attribut „enhanced“ für das MBSR-Programm ableiten. Die bestehende medikamentöse Behandlung (Prophylaxe sowie Akutmedikation) sollte unverändert weitergeführt werden.

Als primäres klinisches Outcome-Maß wurde die Veränderung der Kopfschmerztage von „Prä“ (Baseline) zu Woche 20 (=Interventionsende) definiert (Erfassung jeweils mit einem täglichen elektronischen Tagebuch über einen Zeitraum von 28 Tagen). Als sekundäre klinische Outcome-Maße wurden die kopfschmerzbedingte Beeinträchtigung (erfasst mit dem HIT-6), die durchschnittliche Kopfschmerzintensität, die Responder-Rate ( $\geq 50 \%$ Reduktion der Kopfschmerztage von Prä zu Woche 20) und die Anzahl der Migränetage bestimmt. Alle Parameter (ausgenommen die Response-Rate) wurden außerdem zu Woche 10 (= Zwischenbilanz in der laufenden Therapie) und Woche 52 (=1-Jahres-Follow-up) erhoben. Darüber hinaus wurden zur Darstellung potenzieller struktureller und funktioneller Veränderungen des Gehirns verschiedene Verfahren der Magnetresonanztomografie (MRT) angewendet. Auch hier wurde zwischen primären Outcome-Maßen (u.a. Aktivierung des linken dorsolateralen präfrontalen Kortex während einer kognitiven Aufgabe sowie Volumen der grauen Substanz in bestimmten Gehirnregionen) und sekundären Outcome-Maßen (Gesamtvolumen der grauen Substanz, Aktivierung durch Schmerzreize, Resting State Konnektivität der Insula) unterschieden.

Mit dem MBSR-Programm konnte eine signifikant höhere Reduktion der Kopfschmerztage zu Woche 10 sowie Woche 20 erzielt werden. So sank die Anzahl der Kopfschmerztage in der MBSR-Bedingung von durchschnittlich 7,8 (Baseline) auf 5,5 (Woche 10) bzw. 4,6 (Woche 20), während die Kopfschmerztage in der SMH-Bedingung von 7,7 (Baseline) nur auf 6,9 (Woche 10) bzw. 6,0 (Woche 20) sanken. Im 52-Wochen-Follow-up kam es allerdings zu einer gewissen Annäherung der Kopfschmerztage (SMH: 5,6; MBRS: 4,6), sodass der Unterschied nicht mehr signifikant war. Bei den sekundären klinischen Outcome-Maßen zeigten sich signifikante Unterschiede im Sinne einer Überlegenheit des MBSR im HIT-6 (Woche 20), in der Responder-Rate nach Woche 20 (SMH: $23 \%$; MBSR $52 \%$ ) und in den Migränetagen (Woche 10 und 20). Auf der Ebene der Bildgebung (MRT) konnten für die hier definierten primären Outcome-Maße (u. a. Aktivierung des linken dorsolateralen präfrontalen Kortex) keine Unterschiede zwischen den Interventionen beobachtet werden. Für die in diesem Kontext erfassten sekundären Parameter ergaben sich jedoch durchaus Unterschiede: Hier zeigte sich für die MBSR-Bedingung signifikant geringere Aktivierung in bestimmten Gehirnregionen bzw. bei bestimmten Aktivitäten, was von den Autoren als positiver Effekt dieser Intervention (im Sinne einer verbesserten „kognitiven Effizienz“) gewertet wird. Ähnliche Befunde liegen auch aus anderen Studien zu Effekten meditativer Verfahren vor.

Insgesamt zeigte sich das MBSR-Programm sowohl in einigen klinischen Parametern 
(v.a. Anzahl der Kopfschmerztage) als auch hinsichtlich bestimmter funktioneller Veränderungen des Gehirns (hier: geringere Aktivierung) im Vergleich mit einer aktiven Kontrollgruppe (SMH) überlegen. Die Autoren kommen daher zu dem Schluss, dass MBSR eine effektive Behandlungsoption zur Migräneprophylaxe ist.

\section{Kommentar}

Die Evidenz von verhaltenstherapeutischen Verfahren zur Migräneprophylaxe kann als gesichert angesehen werden. Allerdings ist nach wie vor unklar, welches verhaltenstherapeutische Verfahren bei welchem Migräne-Patienten wie und in welchem Ausmaß wirkt. Auch ist die Facette der achtsamkeitsbasierten Verfahren, die inzwischen einen wichtigen Bereich verhaltenstherapeutischer Interventionen darstellen, im Kontext der Migräneprophylaxe kaum untersucht. Von daher ist die vorliegende Studie ein wichtiger Beitrag zum Kenntnisstand über verhaltenstherapeutische Therapieoptio- nen bei Migräne. Es konnte gezeigt werden, dass ein achtsamkeitsbasiertes Therapieprogramm (MBSR) eine ernstzunehmende Alternative zu traditionellen verhaltenstherapeutischen Interventionen (Stressbewältigung) ist. Eine Stärke der Studie ist der für psychotherapeutische Interventionsstudien vergleichsweise große Stichprobenumfang. Des Weiteren imponiert der Ansatz, neben klinischen Parametern auch bildgebende Verfahren (MRT) zur Effektivitätsbeurteilung heran zu ziehen. Profitiert hätte die Studie noch von der Angabe von Effektstärken. Die Unterteilung in primäre und sekundäre Outcome-Maße wirkt zum Teil etwas sehr willkürlich. So erschließt sich u.a. nicht, warum die Messung von Woche 20 einen höheren Stellenwert als die von Woche 52 (in welcher die Maßnahmen ggf. erst ihre volle Wirksamkeit entfalten können) haben soll. Hilfreich wäre außerdem die Berechnung einer Responder-Rate für alle klinischen Outcome-Maße gewesen. Abgesehen von diesen kleinen statistisch-methodischen Schwächen kann die Aussage- kraft der Ergebnisse als hoch bewertet werden. Die Studie liefert somit einen wichtigen Impuls, achtsamkeitsbasierte Verfahren in der Migräneprophylaxe zukünftig stärker zu berücksichtigen.

Timo Klan, Mainz

\section{Literatur}

[1] Kabat-Zinn J. Full catastrophe living: The program of the Stress Reduction Clinic at the University of Massachusetts Medical Center. New York: Delta; 1990

[2] Segal ZV, Williams JMG, Teasdale JD. Mindfulness-based cognitive therapy for depression: a new approach to preventing relapse. New York: Guilford Press; 2002

[3] Heidenreich T, Michalak J, Eifert G. Balance von Veränderung und achtsamer Akzeptanz: Die dritte Welle der Verhaltenstherapie. Psychother Psychosom Med Psychol 2007; 57: 475-83

\section{Patienten mit Nackenschmerzen während einer Migräneattacke haben auch außerhalb der Attacken eine erhöhte Muskelspannung in der Nackenmuskulatur}

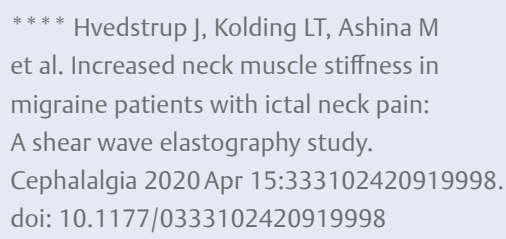

Interiktale Veränderungen der Nackenmuskulatur tragen zu der Schmerzlokalisation während einer Migräneattacke bei.

\section{Hintergrund}

Kopfschmerz-Patienten mit Spannungskopfschmerzen und Migräne geben häufig Nackenschmerzen sowohl iktal als auch interiktal an. Ob diese Schmerzen die Ursache oder Folge von Migräneschmerzen sind, ist nicht abschließend geklärt. Eine entsprechende Zuordnung würde therapeutische Verfahren im Bereiche des Nackens als mögliche kausale Therapie unterstützen. Die Autoren untersuchten diesen Zusammenhang mittels Bestimmung der Muskelsteifigkeit (Nacken und M. trapezius) sowie der Druckschmerzschwellen.

\section{Zusammenfassung}

Die dänische Arbeitsgruppe beschäftigt sich seit einiger Zeit mit der Frage, inwieweit nozizeptive Aktivität aus der Muskulatur zum Migränekopfschmerz beiträgt. In der vorgelegten Arbeit wurden 100 Patienten mit einer episodischen Migräne mit oder ohne Aura und 46 Kontrollen untersucht. 52 der Migräne-Patienten berichteten über iktale Nackenschmerzen, interiktale Nackenschmerzen fanden sich bei 46-48\% der Migräne-Patienten aber auch $30 \%$ der Kontrollen. Ausschlusskriterien waren Verletzungen der HWS, andere Muskelschmerzen bzw. sekundäre Kopfschmerzen. Spannungskopfschmerzen waren erlaubt. Patienten wurden außerhalb der Attacke und ohne begleitende Schmerzmedikation untersucht. Die Untersuchung bestand in der Bestimmung der Fortpflanzungsgeschwindigkeit eines UItraschallsignales in der Muskulatur mittels B-Bild Muskelsonografie (9MHz Reiz, Aufnahme mittels eines GE-Gerätes). Dabei gilt, umso steifer das Gewebe ist, umso schneller pflanzt sich die Stoßwelle im Gewebe fort.
Jede Messung bestand aus 8 Einzelmessungen jeweils für den re./li. M. trapezius sowie den M. splenius capitis und M. semispinalis capitis. Daneben wurde ähnlich zur quantitativen sensorischen Testung (QST) die Druckschmerzschwellen in diesen Arealen sowie im Bereich des M. temporalis erhoben. In der statischen Auswertung wurde für die Faktoren BMI, Geschlecht und Alter adjustiert.

\section{Ergebnisse}

Migräne-Patienten mit iktalen Nackenschmerzen hatten eine höhere Muskelsteifigkeit in den Nackenmuskeln (M splenius capitis und M. semispinalis capitis, nicht im M. trapezius) als Patienten ohne iktale Nackenschmerzen.

Überraschend fand sich eine Korrelation der Attackenhäufigkeit mit der Steifigkeit im M. trapezius. Patienten mit einer Migräne und ohne iktale Nackenschmerzen unterschieden sich nicht von den Kontrollen. 
Patienten mit interiktalen Nackenschmerzen unterschieden sich nicht von denen ohne interiktale Schmerzen. Die Muskelsteifheit in allen Arealen zeigte eine Altersabhängigkeit.

Für die Druckschmerzschwellen ergaben sich keine Unterschiede zwischen den beiden Patientengruppen mit Migräne (mit und ohne Nackenschmerzen) und zwar sowohl für die Nackenmuskeln, dem M. trapezius als auch dem M. temporalis. Die Schwellen waren an all diesen Punkten gegenüber den Kontrollen erniedrigt.

Die Autoren interpretieren die Daten dahin, dass es keinen Hinweis darauf gibt, dass eine primäre zentrale Sensitivierung Ursache der unterschiedlichen Steifigkeit ist, da die Druckschmerzschwellen innerhalb der Migränegruppen nicht verschieden sind. Die Daten werden vielmehr als Hinweis auf eine periphere Ursache für die vermehrte Steifigkeit gesehen, einerseits als Folge einer Inflammation der Muskulatur (z. B. vermehrte IL6-Freisetzung) und dadurch vermehrte Aktivierung von Nozizeptoren aber auch einer vermehrten Muskelanspannung, bzw. Muskelumbau, und andererseits Ausbildung von muskulären Triggerpunkten (wie sie möglicherweise auch bildgebend dargestellt werden können [1]).

\section{Kommentar}

Die Bedeutung der perikraniellen Muskulatur in der Schmerzentstehung bei der Migräne ist zuletzt wieder mehr in den Mittelpunkt gerückt, auch durch den Effekt von Botulinumtoxin. Die vorgestellte Arbeit verwendet eine Methode, die noch nicht weit verbreitet ist und auf die Darstellung einer durch einen Ultraschallimpuls ausgelösten Welle in der Muskulatur beruht. Untersuchungen mit dem Ultraschallgerät sind aber doch in bestimmten Maße Untersucher abhängig, was auch die hohe Tag-zu-Tag-Variabilität, die die Autoren diskutieren, mit erklären kann. Weiter ist zu beachten, dass bei der Untersuchung der Nackenmuskel regelmäßig 2 Muskeln, die in Faszien eingehüllt sind, untersucht wurden. Die Autoren gehen nicht darauf ein, wie dieses die Messung beeinflussen kann. Man würde erwar- ten, dass neben der Attackenhäufigkeit interiktale Nackenschmerzen das Ergebnis (Steifigkeit der Nackenmusken) beeinflussen, was aber nicht gefunden wurde. Insgesamt sind die Ergebnisse interessant und sollten zu einer weiteren Untersuchung der Rolle der perikraniellen Muskulatur bei der Migräne führen, sind aber noch zu inkonklusiv, um daraus endgültige Schlüsse zu ziehen.

Andreas Straube, München

\section{Literatur}

[1] Landgraf MN, Ertl-Wagner B, Koerte IK et al. Alterations in the trapezius muscle in young patients with migraine - a pilot case series with MR. Eur J Paediatr Neurol 2015; 19 (3): $372-6$

\section{Modulation der Aktivität von trigeminalen Nervenfasern durch CGRP}

\begin{abstract}
$* * * *$ Jacob C. A. Edvinsson, Karin Warfvinge et al. C-fibers may modulate adjacent $A \delta$-fibers through axon-axon CGRP signaling at nodes of Ranvier in the trigeminal system. The Journal of Headache and Pain 2019; 20: 105. doi.org/10.1186/s10194019-1055
\end{abstract}

CGRP aus C-Fasern könnte auf die Schnürringe benachbarter $A \beta$-Fasern einwirken eine neue Hypothese zur peripheren Wirkung von CGRP.

\section{Hintergrund}

Dachte man früher, dass das Neuropeptid Calcitonin gene-related peptide, neben seiner Wirkung als Vasodilatator bei der neurogenen Entzündung und als Neuromodulator im spinalen Hinterhorn, lediglich ein Bioindikator für die starke trigeminale Aktivität bei Migräne und Clusterkopfschmerz ist, so glaubt man inzwischen, dass es durchaus auch eine verstärkende Signalwirkung im peripheren Nervensystem haben kann [1]. Es kann zwar keine nozizeptiven Neurone direkt erregen und ist auch nicht schmerzhaft, wenn es injiziert wird, aber es kann bei Migräne- und Clusterkopfschmerz-Patienten nach einer Verzögerung von bis zu mehreren Stunden migräne- bzw. clusterartige Attacken ausIösen [2]. Umgekehrt hat die Hemmung des CGRP-Signalsystems durch CGRP-Rezeptorantagonisten (Gepante) oder durch monoklonale Antikörper einen klaren therapeutischen Effekt bei Migräne [3]. Für beide Substanzgruppen ist eine zentrale Wirkung aber unwahrscheinlich, da sie kaum die Blut-Hirnschranke überwinden können [4]. Deshalb wird seit einiger Zeit heftig darüber diskutiert, wo und wie CGRP im peripheren trigeminalen System wirkt. Dafür kommen eigentlich nur das Ganglion trigeminale und die peripheren Fortsätze der Ganglienzellen in Frage, vorwiegend solche, welche die Hirnhäute (speziell Dura mater encephali) innervieren, denn dieses Gewebe gilt als der bevorzugte Ort der primär-afferenten Aktivierung bei der Kopfschmerzentstehung [5].

\section{Zusammenfassung}

Immunhistochemische Untersuchungen zeigten, dass im Ganglion trigeminale 2 Neuronentypen vorkommen, die einen bilden CGRP, die anderen exprimieren CGRP-Rezeptorproteine wie CLR und RAMP1. Es wird angenommen, dass die CGRP-bildenden Neurone unmyelinisierte C-Fasern ausbilden, während die CGRP-Rezeptorbildenden Neurone dünn myelinisierte $A \beta$-Fasern als Fortsätze haben. Beide Fasertypen konnten in der Dura mater encephali beobachtet werden. Bei der Doppel-Immunfärbung gegen CASPR, einem Marker für die paranodale Region von Ranvierschen Schnürringen, und gegen das CGRP-Rezeptorprotein RAMP1 oder ein Fusionsprotein für CGRP-Rezeptoren (AA58) fiel auf, dass die Marker kolokalisiert waren und sich manchmal in direkter Nähe von CGRP-immunpositiven Verdickungen fanden. Dies ließ die Schlussfolgerung zu, dass freigesetztes CGRP auf die Ranvierschen Schnürringe der $A \beta$-Fasern wirken könnte, 
in denen immunhistochemisch zudem Proteinkinase A nachgewiesen werden konnte, welche bekanntermaßen durch cAMP aktiviert werden kann. Aus diesen immunhistochemischen Befunden wurde geschlossen, dass CGRP aus den C-Fasern an der axonalen Membran in den Ranvierschen Schnürringen der $A \beta$-Fasern wirkt, indem es dort über die Bindung an CGRP-Rezeptoren und der bekannten intrazellulären Signalkaskade die Proteinkinase A aktiviert und eine Sensibilisierung von Kanalproteinen bewirkt. Diese Interaktion könnte somit auch der periphere Wirkungsort von Gepanten und monoklonalen Antikörpern sein, die diese Signalwirkung unterbrechen.

\section{Kommentar}

Diese neue Hypothese, welche in der kommentierten Arbeit mit dem Erstautor Jacob Edvinsson, dem Sohn von Lars Edvinsson, vorgestellt wurde, entbehrt nicht einer gewissen Genialität. Allerdings beruht sie alleine auf immunhistochemischen Untersuchungen und ist ohne funktionellen Nachweis, was vielleicht den Gutachtern aufgefallen sein mag, weshalb die Arbeit möglicherweise nicht höherrangig publiziert wurde. Sie hat darüber hinaus mehrere Schwächen und Widersprüche, die im Folgenden diskutiert werden sollen.

- Der paranodale Marker (CASPR) und die Marker für CGRP-Rezeptoren wurden als kolokalisiert erkannt, aber da die mikroskopische Analyse weder mit konfokaler Mikroskopie noch mit elektronenmikroskopischen Techniken durchgeführt wurde, ist eine genaue Zuordnung dieser Marker zu zellulären Strukturen gar nicht möglich. Es besteht nämlich durchaus Uneinigkeit darüber, welche Zellen die peripheren CGRP-Rezeptoren exprimieren. Nach [6] sind sie nicht in der Axonmembran, sondern in der peripheren Glia, den Schwannzellen exprimiert, aber nicht in peripheren Axonen.

- Die in dieser Arbeit diskutierte Hypothese stützt sich auf funktionelle Befunde aus der Arbeitsgruppe von R. Burstein in Boston, welche aufgrund von 2 elektrophysiologischen Untersuchungen [7, 8] zum Ergebnis kamen, dass CGRP aus den C-Fasern in der Dura mater auf $A \beta$-Fasern aktivierend wirkt und dass die Neurotransmis- sion nozizeptiver Signale im spinalen Trigeminuskern selektiv durch A $\beta$-Faser-Eingänge erfolgt. (Diese Arbeiten wurden bereits in einer der letzten Kopfschmerz-News diskutiert). Die Eindeutigkeit der Ergebnisse der Burstein-Gruppe steht allerdings in Frage und kann aus zellanatomischen Gründen nicht in ihrer strikten Form aufrechterhalten werden, da nur etwa die Hälfte der C-Faser-Neurone überhaupt CGRP bildet, während ein nicht unerheblicher Teil der $A \beta$-Neurone ebenfalls CGRP-bildend ist.

- Sollten CGRP-Rezeptoren tatsächlich in der Axonmembran von $A \beta$-Fasern vorhanden sein und es bei Bindung von CGRP über CAMP zu einer Aktivierung der Proteinkinase A kommen, stellt sich die Frage, ob eine Phosphorylierung von lonenkanälen, wie sie in der Arbeit (zumindest in den schönen grafischen Abbildungen) angedeutet wird, überhaupt zu Sensibilisierungsvorgängen führen kann. Wenn die paranodalen Kaliumkanäle phosphoryliert und damit leitfähiger werden, müsste das zu einer verstärkten Repolarisation und damit eher zu abgeschwächten Aktionspotenzialen führen. Diese Wirkung ist ja bei glatten Muskelfasern bekannt, wo CGRP über die Aktivierung von Kaliumkanälen durch Hyperpolarisation zur Relaxation führt [9]. Sollten aber die nodalen spannungsabhängigen Natrumkanäle phosphoryliert und damit leitfähiger werden, würde das bestenfalls zur Vergrößerung von Aktionspotentialen führen und damit zu einem verstärkten Elektrotonus (der dann möglicherweise mehr Schnürringe erreicht und die Nervenleitungsgeschwindigkeit erhöht). Da aber die Signalstärke eines sensorischen Neurons über das Axon alleine durch die Frequenz (oder Anzahl) der Aktionspotenziale vermittelt wird, ist die Größe der Aktionspotenziale unerheblich.

- Die postulierten Phosphorylierungsvorgänge laufen innerhalb von Sekunden ab, die CGRP-Wirkungen sind aber verzögert. Der langsame Zeitverlauf spricht eher für einen modulierenden Effekt auf Genexpressionsvorgänge, die aber neuronal allesamt in den Zellkörpern, also im Ganglion trigeminale, stattfinden und ihre Wirkungen in der peripheren Nervenfaser erst nach axonalem Transport z. B. von Kanalproteinen, entfalten können.

- Trotz dieser Widersprüche ist die vorliegende Arbeit von Bedeutung, denn es könnte sich auch um eine Signalwirkung des CGRP auf die Schwannzellen handeln, die ebenfalls CGRP-Rezeptoren exprimieren können [6]. Eine erhöhte Leitfähigkeit von Kaliumkanälen könnte z. B. durch die Pufferfunktion dieser Gliazellen die extrazelluläre Kaliumkonzentration herabsetzen, was bekanntermaßen zur Depolarisation von erregbaren Membranen und Hyperexzitabilität führt.

- Solche Szenarien könnten sich genauso gut im Ganglion trigeminale abspielen, z. B. auch zwischen Neuronen und Satellitenzellen, welche ebenfalls CGRP-Rezeptoren bilden können. Die vorliegende Arbeit ist somit ein guter Ausgangspunkt, um mehr über die Beziehung zwischen Neuronen und Gliazellen bezüglich freigesetzter Neuropeptide wie dem CGRP zu erforschen.

\section{Karl Meßlinger, Erlangen}

Literatur

[1] Russo AF. Calcitonin gene-related peptide (CGRP): a new target for migraine. Annu Rev Pharmacol Toxicol 2015; 55: 533-52

[2] Hansen JM, Hauge AW, Olesen J et al. Calcitonin gene-related peptide triggers migraine-like attacks in patients with migraine with aura. Cephalalgia 2010; 30 (10): 1179-86

[3] Charles A, Pozo-Rosich P. Targeting calcitonin gene-related peptide: a new era in migraine therapy. Lancet 2019; 394 (10210): 1765-74

[4] Edvinsson L, Warfvinge K. Recognizing the role of CGRP and CGRP receptors in migraine and its treatment. Cephalalgia 2019; 39 (3): 366-73

[5] Olesen J, Burstein R, Ashina M et al. Origin of pain in migraine: evidence for peripheral sensitisation. Lancet Neurol 2009; 8 (7): 679-90

[6] Lennerz JK, Rühle V, Ceppa EP et al. Calcitonin receptor-like receptor (CLR), receptor activity-modifying protein 1 (RAMP1), and calcitonin gene-related peptide (CGRP) immunoreactivity in the rat trigeminovascular system: differences between peripheral and 
central CGRP receptor distribution. I Comp Neurol 2008; 507 (3): 1277-99

[7] Melo-Carrillo A, Noseda R, Nir R et al. Selective Inhibition of Trigeminovascular Neurons by Fremanezumab: A Humanized Monoclonal Anti-CGRP Antibody. J Neurosci Off J Soc Neurosci 2017; 37 (30): 7149-63
[8] Melo-Carrillo A, Strassman AM, Nir R et al. Fremanezumab-A Humanized Monoclonal Anti-CGRP Antibody-Inhibits Thinly Myelinated (Aס) But Not Unmyelinated (C) Meningeal Nociceptors. J Neurosci Off J Soc Neurosci 2017; 37 (44): 10587-96
[9] Russell FA, King R, Smillie S-J et al. Calcitonin gene-related peptide: physiology and pathophysiology. Physiol Rev 2014; 94 (4): 1099-142

\section{Eptinezumab zur Prophylaxe der chronischen Migräne}

\author{
**** Lipton RB, Goadsby PJ, Smith J et al. \\ Efficacy and safety of eptinezumab in patients \\ with chronic migraine. Neurology 2020; 94 : \\ e1365-e1377
}

Der CGPR-Antikörper Eptinezumab ist schnell wirksam und gut verträglich in der Prophylaxe der chronischen Migräne.

\section{Hintergrund}

Monoklonale Antikörper gegen Calcitonin Gene-Related Peptide (CGRP) und seinen Rezeptor sind die ersten Substanzen, die spezifisch zur Migräneprophylaxe entwickelt wurden. In Deutschland sind 3 CGRP-(Rezeptor)-Antikörper auf dem Markt erhältlich: Erenumab, Galcanezumab und Fremanezumab. Der vierte „Migräne-Antikörper“, Eptinezumab, ist erst seit Februar 2020 und nur in den USA mit dem Namen Vyepti ${ }^{\mathrm{TM}}$ zugelassen. Alleinstellungsmerkmal von Eptinezumab ist die intravenöse Verabreichung, wohingegen die anderen CGRP-(Rezeptor)-Antikörper subkutan appliziert werden. Im Folgenden werden die Hauptergebnisse der Phase-III-Studie von Eptinezumab zur Prophylaxe der chronischen Migräne dargestellt.

\section{Zusammenfassung}

Die PROMISE-2-Studie war eine multizentrische, randomisiert-kontrollierte, doppelblinde Studie und umfasste 1121 Patienten mit chronischer Migräne. Diese erhielten entweder Eptinezumab 100 mg, Eptinezumab $300 \mathrm{mg}$ oder Placebo jeweils 2-mal im Abstand von 12 Wochen als intravenöse Kurzinfusion. Eine gleichzeitige orale Migräneprophylaxe in stabiler Dosierung war erlaubt. Primärer Endpunkt der Studie war die Änderung der monatlichen Migränetage zwischen der 4-wöchigen Baseline-Phase vor der ersten Infusion und Woche 1 bis 12 .
Die Studienteilnehmer waren im Durchschnitt 40,5 Jahre alt und zu 88,2\% weiblich. Während der Baseline hatten sie durchschnittlich 16,1 Migränetage. Beide Eptinezumab-Dosierungen führten zu einer signifikanten Reduktion der monatlichen Migränetage in Wochen 1 bis 12 im Vergleich zu Placebo: $-7,7$ unter $100 \mathrm{mg},-8,2$ unter $300 \mathrm{mg}$ und $-5,5$ unter Placebo $(p<0,001$ für beide Gruppen). Eine $50 \%$-Reduktion der monatlichen Migränetage wurde von $57,6 \%$ der Patienten unter 100 mg, 61,4\% unter $300 \mathrm{mg}$ und $39,3 \%$ unter Placebo erreicht ( $p<0,001$ für beide Gruppen vs. Placebo). Bereits am ersten Tag nach der Infusion wurde der Wirkunterschied zwischen Verum und Placebo deutlich: Ungefähr $28 \%$ der Patienten in beiden Eptinezumab-Gruppen entwickelten an diesem Tag eine Migräneattacke verglichen mit 42,3\% in der Placebogruppe $(p<0,001)$. Unter Eptinezumab kam es zudem zu einer signifikanten Reduktion der monatlichen Tage mit Einnahme von Akutmedikation sowie zu einer signifikanten Besserung der kopfschmerzassoziierten Lebensqualität (Headache Impact Test-6).

Die Nebenwirkungsraten in den Eptinezumab-Gruppen und in der Placebogruppe waren vergleichbar. Die häufigsten Nebenwirkungen waren Erschöpfung (1,8\% in den Eptinezumab-Gruppen und $<1 \%$ unter Placebo) und Übelkeit (1,6\% in den Eptinezumab-Gruppen und < 1 \% unter Placebo).

\section{Kommentar}

Die prophylaktische Behandlung von Patienten mit chronischer Migräne stellt häufig eine therapeutische Herausforderung dar. Mit der Einführung monoklonaler Antikörper wurden die möglichen Behandlungsoptionen beträchtlich erweitert, insbesondere für Patienten, die auf traditionelle medikamentöse Prophylaxen nicht ausreichend angesprochen oder Nebenwirkungen entwickelt hatten. Die Behandlung dieser Patienten mit Erenumab, Galcanezumab und Fremanezumab hat sich seit Herbst 2018 in Deutschland gut etabliert. Eptinezumab ist nun der vierte „Migräne-Antikörper“ gegen den CGRP-Signalweg mit positiven Studienergebnissen. Eine baldige europäischen Zulassung vorausgesetzt, stellt sich die Frage, welchen Platz im Arsenal der Migränetherapeutika Eptinezumab einnehmen wird.

Hauptunterschied zwischen Eptinezumab und den anderen CGRP-(Rezeptor)-Antikörpern ist seine intravenöse Verabreichung. Die anderen Antikörper sind als subkutane Fertigspritzen bzw. -pens erhältlich und ermöglichen somit eine Selbstapplikation durch den Patienten zu Hause. Die Eptinezumab-Gaben setzen hingegen immer einen längeren Kontakt zum medizinischen Personal voraus. Insbesondere Patienten, die eine Selbstapplikation nicht durchführen können oder negative Erfahrungen mit der subkutanen Applikation gemacht haben, könnten von Eptinezumab profitieren. Eine intravenöse Verabreichung geht auch mit einem höheren Placeboeffekt einher: So waren in der PROMISE-2-Studie die Response-Raten in allen Gruppen höher als in vergleichbaren Studien mit anderen CGRP-(Rezeptor)-Antikörpern, die placebosubtrahierten Ergebnisse jedoch vergleichbar. Ein weiterer Vorteil der intravenösen Applikation ist die höhere Bioverfügbarkeit und das schnellere Anfluten der Substanz: Wie in PROMISE-2 beschrieben, kann man eine Wirkung von Eptinezumab bereits nach einem Tag feststellen.

Ein weiteres wichtiges Merkmal von Eptinezumab ist das lange Applikationsintervall von 12 Wochen. Bisher ist nur Fremanezumab in 3-monatlichen Intervallen applizierbar, während Erenumab und Galcanezumab 
alle 4 Wochen verabreicht werden. Längere Therapieintervalle könnten dazu beitragen, dass eine prophylaktische Medikation besser angenommen und dadurch eine bessere Therapieadhärenz erreicht wird.

Zusammenfassend stellt Eptinezumab eine weitere wirksame Alternative zur Prophyla- xe der chronischen Migräne dar. Durch die intravenöse Applikation und das längere Applikationsintervall werden die Behandlungsalternativen mit monoklonalen Antikörpern erweitert und individuelle Behandlungswünsche können besser berücksichtigt werden. Die Nutzung von Eptinezumab in der klinischen Praxis wird zeigen, ob dies eine tatsächliche Erweiterung unserer Möglichkeiten zur Migräneprophylaxe darstellt und welche Patienten von dieser Therapie besonders profitieren können.

Bianca Raffaelli, Berlin

\section{OnabotulinumtoxinA zur Behandlung der chronischen Migräne bei Jugendlichen}

** Winner PK, Kabbouche M, Yonker M, et al. A Randomized Trial to Evaluate OnabotulinumtoxinA for Prevention of Headaches in Adolescents With Chronic Migraine. Headache 2020; 60 : 564-575

OnabotulinumtoxinA ist in der Prophylaxe von Kopfschmerzen bei Jugendlichen mit chronischer Migräne nicht wirksamer als Placebo.

\section{Hintergrund}

Randomisierte kontrollierte Studien zur medikamentösen Therapie der Migräne bei Kindern und Jugendlichen stellen aufgrund der hohen Ansprechrate auf Placebo eine besondere Herausforderung dar. Dies trifft auf die Akuttherapie [1] genauso zu wie auf die Prophylaxe [2]. Eine Reihe von Studien zur Wirksamkeit der Triptane zeigte bei Kindern und Jugendlichen zwar ein mit Erwachsenen vergleichbares Ansprechen auf die aktive Substanz aber keinen signifikanten Unterschied zu Placebo. Daher wurde in späteren Studien ein modifiziertes Studiendesign eingesetzt, bei dem in einer Eingangsphase alle Teilnehmer Placebo erhielten und nur jene in die eigentliche Studie aufgenommen wurden, die kein Ansprechen auf Placebo zeigten [1].

Unter den Studien zur medikamentösen Migräneprophylaxe bewertet die Leitlinie der Deutschen Gesellschaft für Neurologie einzig die Wirksamkeit von Flunarizin als gesichert, was auch der Einschätzung der Verfasserin entspricht. Valproinsäure ist nicht wirksam. Eine Studie zu Topiramat und Amitriptylin mit einer sehr großen Zahl geplanter Teilnehmer wurde aufgrund einer sehr hohen Placeborate vorzeitig abgebrochen. Der primäre Endpunkt wurde unter Placebo, Topiramat und Amitriptylin in $61 \%$, $55 \%$ und $52 \%$ erreicht.
Prinzipiell stellt sich zudem die Frage, bei welchen Kindern und Jugendlichen eine medikamentöse Migräneprophylaxe überhaupt erforderlich ist. Vor diesem Hintergrund ist es verständlich, dass die Herausforderungen an die besprochene erste randomisierte kontrollierte Studie zur Wirksamkeit und Verträglichkeit von OnabotulinumtoxinA bei Jugendlichen mit chronischer Migräne sehr hoch waren.

\section{Zusammenfassung}

Winner et al. haben in einer multizentrischen, doppelblinden, randomisierten $\mathrm{Pa}$ rallelgruppenstudie eine einzelne Behandlung mit OnabotulinumtoxinA bewertet. Es wurde eine Dosis von 155 oder 74 Einheiten OnabotulinumtoxinA nach dem „fixed site, fixed dose“ Schema intramuskulär verabreicht und mit Placebo (Kochsalzlösung) verglichen. Primärer Endpunkt war die Änderung der Häufigkeit der Kopfschmerztage in den Wochen 9 bis 12 gegenüber der Baseline. Weitere Endpunkte waren die Änderung der Häufigkeit von Kopfschmerztagen in den Wochen 1 bis 4 und 5 bis 8 und die Änderung der Häufigkeit schwerer Kopfschmerzen. Zudem wurden die Sicherheit und Verträglichkeit bewertet.

Von 125 randomisierten Patienten wurden 45 mit 155 Einheiten OnabotulinumtoxinA, behandelt, 43 erhielten 74 Einheiten und 37 erhielten Placebo. Alle Teilnehmer wurden in die primäre Wirksamkeitsanalyse einbezogen, und 115 (92,0\%) beendeten die Studie. 4 Patienten haben die Studie wegen mangelnder Wirksamkeit abgebrochen, niemand wegen unerwünschter Ereignisse. In allen 3 Studienarmen zeigte sich eine Reduktion der Kopfschmerztage in den Wochen 9 bis 12 ohne signifikante Unterschiede zwischen den Behandlungen. Die mittlere Re- duktion lag zwischen 6,3 Tagen (155 Einheiten OnabolutinumtoxinA) und 6,8 Tagen (Placebo). Alle Behandlungen reduzierten die Häufigkeit schwerer Kopfschmerztage und wurden gut vertragen.

\section{Kommentar}

Der Erstautor ist sehr erfahren in der Durchführung von Studien zur medikamentösen Therapie der Migräne bei Kindern und Jugendlichen. In die Studie wurden Jugendliche eingeschlossen, bei denen eine chronische Migräne im Durchschnitt seit 4,2 Jahren bestand. Im Artikel finden sich keine Informationen über Vortherapien. Andere wichtige Limitationen werden in der Diskussion kritisch angeführt, nämlich die geringe Zahl an Teilnehmern, die (zu) kurze Studiendauer und die nur einmalige Verabreichung der Studienmedikation. Auch das Problem der hohen Placebo-ResponderRaten wird diskutiert. Künftige Studien zur Pharmakoprophylaxe der Migräne, sollten einerseits auf Patienten beschränkt sein, bei den vordefinierte nicht pharmakologische Maßnahmen nachgewiesenermaßen nicht wirksam waren und andererseits eine Studieneingangsphase enthalten, die das Ziel hat, Placebo-Responder auszuschließen.

Berücksichtigt man die Verabreichung von OnabotulinumtoxinA an 31 Injektionsstellen im Bereich der perikraniellen Muskulatur, wären Studien zur Wirksamkeit und Verträglichkeit von monoklonalen Antikörpern gegen calcitonin gene-related peptide bei Jugendlichen angesichts der wesentlich angenehmeren subkutanen Gabe einmal im Monat eine interessante Alternative für eine streng ausgewählte Gruppe besonders schwer von Migräne betroffener junger Patienten. Der vorrangige Stellenwert 
einer Beratung zu Lebensstil- und Verhaltensfaktoren sowie die Beurteilung und Behandlung von komorbiden Störungen, den die Verfasserin seit langem betont [4], kann nicht deutlich genug hervorgehoben werden $[3,5]$.

\section{Çiçek Wöber-Bingöl, Wien}

\section{Literatur}

[1] Wöber-Bingöl Ç. Pharmacological treatment of acute migraine in adolescents and children. Paediatr Drugs 2013; 15: 235-246

[2] Powers SW et al. Trial of amitriptyline, topiramate, and placebo for pediatric migraine. N Engl J Med 2017; 376 (2): 115-124
[3] Diener HC et al. Therapie der Migräneattacke und Prophylaxe der Migräne. Leitlinie der Deutschen Gesellschaft für Neurologie (DGN) in Zusammenarbeit mit der Deutschen Migräne- und Kopfschmerzgesellschaft (DMKG), 2018. Im Internet: www.dgn.org/leitlinien/3583-II-030-0572018-therapie-der-migraeneattacke-undprophylaxe-der-migraene; Stand: 5.5.2020

[4] Wöber C, Wöber-Bingöl C. Clinical management of young patients presenting with headache. Funct Neurol 2000; 15 Suppl 3: 89-105

[5] Oskoui $\mathrm{M}$ et al. Practice guideline update summary: Pharmacologic treatment for pediatric migraine prevention: Report of the Guideline Development, Dissemination, and Implementation Subcommittee of the American Academy of Neurology and the American Headache Society. Neurology 2019; 93: 500-509

\section{INFORMATION}

***** Exzellente Arbeit, die bahnbrechende Neuerungen beinhaltet oder eine ausgezeichnete Übersicht bietet

**** Gute experimentelle oder klinische Studie

*** Gute Studie mit allerdings etwas geringerem Innovationscharakter

** Studie von geringerem klinischen oder experimentellen Interesse und leichteren methodischen Mängeln

* $\quad$ Studie oder Übersicht mit deutlichen methodischen oder inhaltlichen Mängeln

Die Kopfschmerz-News werden betreut von: Priv.-Doz. Dr. Ruth Ruscheweyh, Klinik und Poliklinik für Neurologie, Klinikum der Universität München, Marchioninistr. 15, 81377 München, Tel. 089/440073907, ruth. ruscheweyh@med.uni-muenchen.de

Die Besprechungen und Bewertungen der Artikel stellen die Einschätzung des jeweiligen Autors dar, nicht eine offizielle Bewertung durch die Deutsche Migräne- und Kopfschmerzgesellschaft. 